\title{
Statistical Analysis of MIMO Scheme under Nakagami Fading Channels
}

\author{
Nagesh K. N *, Satyanarayana D**, Madhava Prabhu S* and M.N Giri Prasad ${ }^{* * *}$ \\ *Middle East College, Knowledge Oasis, Al Rusyal, Sultanate of Oman \\ ${ }^{* *}$ ECE Department, RGMCET, Nandyal- 528502, A.P, India. \\ ${ }^{* * *}$ ECE Department, JNTUCE, Anantapur- 515002, A.P, India. \\ nagesh@mec.edu.om, madhav@mec.edu.om
}

\begin{abstract}
Node isolation is one of the fundamental metrics in the design consideration and evaluation of wireless ad hoc network. This paper is aimed to introduce a statistical framework for the computing of the isolation probability of a randomly chosen node in a wireless network. The statistical framework is proposed in the context of lognormal shadowing on Nakagami fading for multiple inputs multiple output schemes. In this work, the focus is on path loss component, network topology, node density and performance of a node. The performance of connectivity between nodes in a dense network are analysed by deriving analytical models. Analysis of experimental results indicate that they are useful in estimating connectivity issues and implementing more secure wireless ad hoc networks.
\end{abstract}

Keywords - Nakagami fading, Multiple Input Multiple Output, Isolated Node, Deterministic channel, lognormal shadowing.

\section{INTRODUCTION}

The network which doesn't rely on preexisting infrastructure is usually called as ad hoc network [1], in which the individual nodes have got more responsibility. This area is one of the highly appreciated fields amongst researchers since last few decades. Connectivity is one of the issues [25] since few decades in such networks [7, 18-20]. Since all the nodes are free to move randomly in any time, the issues of connectivity arise in an ad hoc network. It is challenging to establish a wellconnected network. It is slightly difficult, as there are chances that a node may leave the network any time [11]. The node hopping property (changing topology with time) causes the problem in establishing a connection between pair of nodes resulting in node isolation. The definition of probability of node isolation can be stated as the failure of a node to establish a connection with other node in the network [10]. A node which is isolated has a crucial part in the proper functioning of the multihop network. Hence a theoretical molding of node isolation will contribute in encasing network connectivity features. The randomness in radio communications is not considered in a simplistic model of channel propagation on which most of the literatures still rely. Over the last few years the considerable attention [24] was given to the aspects of the connection issues and the area coverage in an adhoc sensor networks of wireless type. To obtain a well-connected network, the problems associated with searching for the transmission range with a necessary and sufficient scaling is discussed in [6]. Penrose has obtained similar results in a more abstract network [7]. In [8], investigation was carried out on scaling for the case of sparse networks. The issues of achieving connectivity in a network of mobile nodes were first addressed in [9]. Bettstetter and Hartmann [10-11] are the first to high light the impact of lognormal shadowing on connectivity of ad-hoc networks. A numerical method for the evaluation of the impact of lognormal shadowing was proposed with qualitative approach. A closed-form expression of such cases was provided in [12]. Haennggi[15] has studied Rayleigh fading impact on network connectivity. These results can be viewed as derived cases of the general case analyzed in [12] for the first time. An ergodic sum rate of multipleinput multiple-output (MIMO) Nakagami-m fading channels with linear minimum mean square error (MMSE) receivers is discussed in [21], [22] and [23]. 
From the literature it is clear that Statistical Analysis of MIMO Scheme has not been explored in the contest Nakagami Fading Channels. It is analyzed on node isolation probability for Deterministic channel, Rayleigh fading channel and lognormal shadowing channel.

The subsequent part of the paper is organized as follows. Section 2 deals with the preliminary assumptions and the system model. A brief analytical evaluation of frame reception rate probability is discussed in Section 3. The numerical and simulation results are described in Section 4 and the same is concluded in Section 5.

\section{SYSTEM MODEL}

Let us consider a wireless ad hoc network, in which the nodes are scattered according to homogeneous Poisson distribution with a parameter $\lambda$ ranging from 0 to $\infty$. Nodes are spread randomly and uniformly over the entire network and independent of each other for complete spatial randomness. Here $\lambda$ is rate of a Poisson random process over a geographical space $\mathfrak{R} 2$.

Let $\mathrm{A}$ be a subset of $\mathfrak{R} 2$, and the network is said to be well connected if there exists at least one connecting path for every pair of node. In disjoint areas, the number of nodes can be seen as independent random variables. In A, the network formed by the nodes is said to be linked if and only if the path exists between each pair of nodes in A. As in [8], the impact of interference from other nodes is assumed as negligible.

Let $P_{I}$ be the probability of node isolation with which a node is isolated in the network. The radio link is assumed Boolean also known as a switched link. With the fading of small scale and lognormal shadowing let $R$ is the random variable with cumulative distribution function (CDF). Where $R$ is non-negative element and given $E\left[R^{2}\right]=$ $\int_{0}^{\infty} 2 \rho d \rho F_{R}^{c}(\rho)$ where $F_{R}^{c}(\rho)$ complimentary cumulative distribution function.

The probability of node isolation is given by [8, 14]

$P_{I}=e^{-\lambda \pi E\left[R^{2}\right]}$

\section{Lognormal Shadowing Model and Path-Loss Model}

Let $P_{t x}$ be the fixed power level at which nodes are assumed to transmit the power. Similarly, $\mathrm{W}$ be the collective white noise power at the receiver. When the average of path loss in lognormal shadowing is described by $K \rho^{-\alpha}$ where $\rho$ is the separation between transmitter and receiver and $\alpha$ is path loss exponent and $k$ is a constant with the path loss model. The received signal to noise power ratio for this channel model is given by $\gamma(\rho)=\left(P_{t x} l(\rho) / W\right)$ where $l(\rho)$ is path loss with reference transmitter and receiver. The communication range $R$ can be calculated using the amount of space between transmitter and receiver for which the SNR fall below the threshold $\psi$. Where $E\left[R^{2}\right]$ and $F_{R}(\rho)$ are given by [13]:

$$
\begin{aligned}
& F_{R}(\rho)=P[\gamma(\rho) \leq \psi]=P\left[l(\rho) \leq \frac{W_{\psi}}{P_{t x}}\right]=1- \\
& \int_{\frac{w \psi}{P_{t x}}}^{\infty} f_{l / r}(a / \rho) d a \\
& E\left[R^{2}\right]=\int_{0}^{\infty} 2 \rho d \rho \int_{\frac{w_{\psi}}{P_{t x}}}^{\infty} f_{l / r}(a / \rho) d a
\end{aligned}
$$

Where $f_{\frac{l}{r}}\left(\frac{\alpha}{\rho}\right)$ represent the PDF of lognormal Shadowing under path loss and given by:

$f_{l / r}(a / \rho)=\frac{1}{\sqrt{2 \pi} \sigma a} e^{-\frac{1}{2}\left[\frac{\ln a-\ln \left(K \rho^{-\alpha}\right)}{\sigma}\right]^{2}}$

\section{A. Lognormal Shadowing and Small Scale Fading}

Large-scale fading is one in which signal attenuation due to signal propagation over large distances and diffraction around large objects in the propagation path. The result of small scale fading considered, let $\gamma$ be the received instantaneous SNR and $y=[\gamma]$, tis average. Given that the transmitter-receiver separation is $\rho$ and the average SNR $y=\left(k P_{t x} \rho^{-\alpha} / W\right)$, let $P_{S}(y)$ is the probability that the receiver instantaneous SNR $\gamma$ with $\operatorname{PDF} f_{l / r}(x / y)$ is greater than a threshold $\psi$, let $P_{S}(y)$ is given by:

$P_{S}(y)=\int_{\Psi}^{\infty} f_{\gamma}(x / y) d x$

Then the quantity $E\left[R^{2}\right]$ is computed as follows:

$E\left[R^{2}\right]=\int_{0}^{\infty} 2 \rho d \rho P_{S}\left[\frac{K P_{t x} \rho^{-\alpha}}{W}\right]$

Consider lognormal shadowing superimposed with small scale, $F_{R}(\rho)$ and $E\left[R^{2}\right]$ are calculated as follows:

$$
\begin{aligned}
& F_{R}(\rho)=1-\int_{0}^{\infty} P_{S}(y) f_{l / r}(a / \rho) d a \\
& E\left[R^{2}\right]=\int_{0}^{\infty} d a \int_{0}^{\infty} 2 \rho d \rho P_{S}\left[\frac{a P_{t x}}{w}\right] f_{l / r}(a / \rho)
\end{aligned}
$$




\section{NODE ISOLATION PROBABILITY FOR NAKAGAMI FADING WITH LOGNORMAL SHADOWING FOR MIMO SCHEME}

The statistical analysis for isolation probability of a node, under nakagami fading with lognormal shadowing in relation with MIMO schemes are derived in the following section.

\section{NAKAGAMI FADING CHANNEL FOR MIMO}

The probability density function of received instantaneous SNR $\gamma$ under the Nakagami-m fading for MIMO scheme is given by [17].

$$
f_{y}(\gamma)=\frac{m^{N_{t} N_{r} m}}{\Gamma\left(N_{t} N_{r} m\right)} \frac{\lambda^{N^{N} N_{r} m-1}}{y^{N_{t} N_{r} m}} e^{\left(-\frac{m \gamma}{y}\right)}
$$

Where,

$N_{t}$ Is number of transmit antennas

$N_{r}$ Is number of receive antennas

$\gamma$ Is instantaneous SNR

$y$ Is average $\mathrm{SNR}$

The success probability $P_{s}(y)=\operatorname{Pr}(\gamma \geq \psi)$ computed as

$P_{S}(y)=\int_{\Psi}^{\infty} \frac{m^{N_{t} N_{r} m}}{\Gamma\left(N_{t} N_{r} m\right)} \frac{\gamma^{N_{t} N_{r} m-1}}{y^{N_{t} N_{r} m}} e^{\left(-\frac{m \gamma}{y}\right)}$

Let $N_{t} N_{r}=M$ after simplification $P_{s}(y)$ computed as in

$P_{S}(y)=e^{-\frac{m \psi}{y}} \sum_{l=0}^{M m-1} \frac{\left(\frac{m \psi}{y}\right) l}{l !}$

$E\left[R^{2}\right]$ can be computed by substituting (11) in (6) in absence of lognormal shadowing as in equation below:

$E\left[R^{2}\right]=\int_{0}^{\infty} 2 \rho d \rho e^{-\frac{m \psi W \rho^{\alpha}}{K P_{t x}}} \sum_{l=0}^{M m-1} \frac{\left(\frac{m \psi W \rho^{\alpha}}{K P}\right)^{l}}{l !}$

$E\left[R^{2}\right]=\sum_{l=0}^{M m-1} \frac{\left(\frac{m \psi w}{K P_{t x}}\right)^{l}}{l !}\left[\left(\frac{2}{\alpha}\right)\left(\frac{m \psi W}{K P_{t x}}\right) \Gamma\left(\frac{2}{\alpha}+l\right)\right]$

Hence the probability of node isolation can be seen as

$P_{I}=$

$\exp \left\{\left(\frac{2}{\alpha}\right)\left(\frac{m \psi W}{K P_{t x}}\right)^{-\left(\frac{2}{\alpha}+l\right)} \Gamma\left(\frac{2}{\alpha}+l\right) \sum_{l=0}^{M m-1} \frac{\left(\frac{m \psi W}{K P_{t x}}\right)^{l}}{l !}\right\}$

\section{A. Nakagami Fading With Superimposed Lognormal Shadowing For MIMO Schemes}

If $y$ is the average SNR of received signal then $E\left[R^{2}\right]$ can be determined by

$E\left[R^{2}\right]=\left(\frac{2}{\alpha}\right)\left(\frac{m \psi W}{K P_{t x}}\right)^{-\frac{2}{\alpha}} e^{\frac{2 \sigma^{2}}{\alpha^{2}}} \sum_{l=0}^{M m-1} \frac{\Gamma\left(\frac{2}{\alpha}+l\right)}{l !}$

Combining equation (1) and (15) the node isolation probability can be determined by:

$$
\begin{aligned}
& P_{I} \\
& =\exp \left\{-\lambda \pi\left[\left(\frac{2}{\alpha}\right)\left(\frac{m \psi W}{K P_{t x}}\right)^{-\frac{2}{\alpha}} e^{\frac{2 \sigma^{2}}{\alpha^{2}}} \sum_{l=0}^{M m-1} \frac{\Gamma\left(\frac{2}{\alpha}+l\right)}{l !}\right]\right\}
\end{aligned}
$$

\section{Numerical And Simulation Results}

This section details the numerical and simulation results obtained using MATLAB tool. The various parameters chosen are $K=10 \mathrm{~dB}$, $P_{t x}=1 \mathrm{~mW}, \psi=1 \mathrm{Watt}$ and $W=0.01 \mathrm{mWatt}$. The parameters like $\lambda, m, \sigma$ and $\alpha$ as assumed suitably. For simulation purpose the network size is selected to be $100 \mathrm{~m} \times 100 \mathrm{~m}$. Based on Poisson process the number of nodes are selected and are placed over the simulation area with reference to uniform distribution. Based on channel modeling links are established. These nodes operate in Nakagami fading event [22] with super imposed lognormal shadowing. The simulation is carried out for isolated node and repeated for many topologies. An average 1000 runs are carried out to determine node isolation probability.

Fig. 1 shows the variation of node isolation probability $P I$ vs. sigma for different value of number of transmit antenna and receive antenna, the result indicates that by increasing the number of antennas the performance will increase or node isolation probability will decrease.fig. 2 plot node isolation probability $P I$ vs. node density for different value of number of antennas for MIMO scheme. From fig. 1 and fig. 2 we can conclude that connectivity can improve by using MIMO scheme or by considering higher value of node density and sigma. Fig.3 plots node density vs. sigma for 
different value of PI, which shows that higher value of node density and sigma will improve the performance. Fig.4 shows impact of path loss exponent $\alpha$ on the isolation probability, keeping all other parameters to be constant. For fixed value of lognormal spread $\sigma$, higher value of path loss exponent always results in larger isolation probability. Hence it could be concluded that large value of lognormal reduces the node isolation.

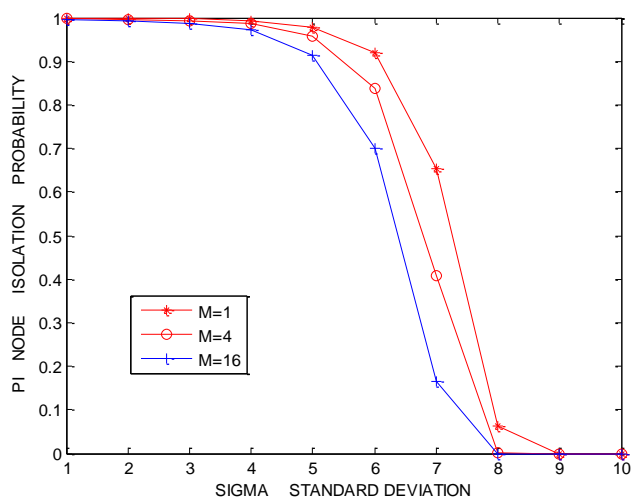

Figure1. Node isolation probability $\mathrm{P}_{I}$ vs. sigma $\sigma$

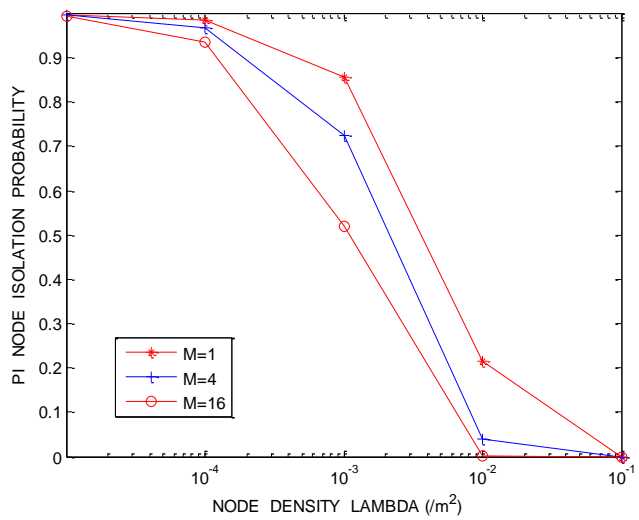

Figure2. Node isolation probability $\mathrm{P}_{I}$ vs. node density $\lambda$

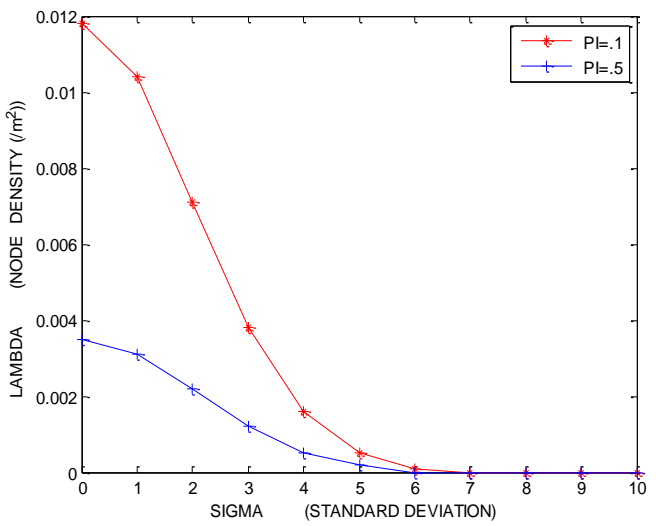

Figure3. Node density $\lambda$ vs. sigma $\sigma$

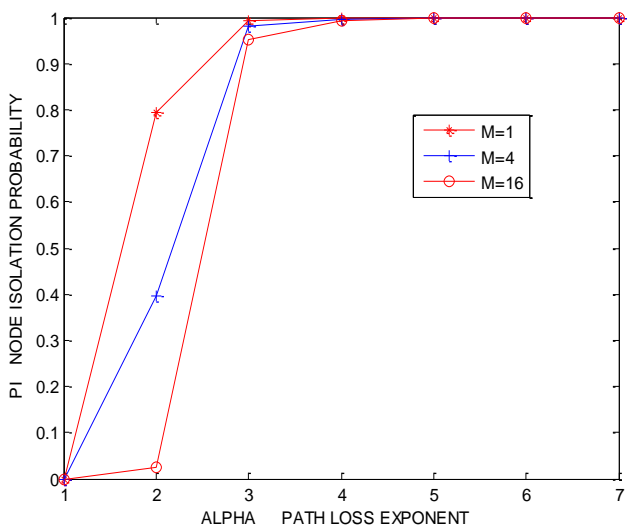

Figure4. Node Isolation Probability $\mathrm{P}_{\mathrm{I}}$ vs. alpha $\alpha$

\section{CONCLUSIONS}

The connectivity between the nodes of a wireless ad-hoc network is one of the important parameter, which needs a lot of work to be carried out from time. It is said to be there in a network only when their deterministic distance is less than transmission radius. Several research work has been done so far considering various parameters of medium as well as fading factor but there is a lack of concentration towards border effect. The effect which concerns about the nodes lying on the boundary region of considerable area of a network is called border effect. This paper analyses the scenario of border effect with several medium conditions. The analysis of node isolation probability considering border effect is done in this research work. As we increase the nodes count in the wireless network, isolation probability of nodes decreasing in the context of border effect, which is opposite scenario to ideal case result. The mathematical analysis provides good understanding of node isolation with border effect. 


\section{REFERENCES}

[1] P. Gupta and P. R. Kumar, Critical power for asymptotic connectivity, IEEE Proc. of CDC, Tamper, FL, USA, 1998.

[2] O. Dousse, P. Thiran, and M. Hasler, Connectivity in ad-hoc and hybrid networks, IEEE Proc. of INFOCOM, New York, USA, 2002.

[3] O. Dousse and P. Thiran, Connectivity vs capacity in dense ad hoc networks, IEEE Proc. of INFOCOM, Hongkong, 2004.

[4] O. Dousse, F. Baccelli and P. Thiran, Impact of interferences on connectivity in ad hoc networks, IEEE/ACM Tran. on Networking, Vol. 13, No. 2, pp. 425 - 436, Apr. 2005.

[5] M. Zorzi and S. Pupolin, Outage probability in multiple access packet radio networks in the presence of fading, IEEE Tran. Veh. Tech., vol. 43, no. 3, pp. 604-610, Aug. 1994.

[6] C. Bettstetter and C. Hartmann, Connectivity of wireless multihop networks in a shadow fading environment, ACM Proc. Of MSWiM, San Diego, CA, 2003.

[7] D. Miorandi and E. Altman, Coverage and connectivity of ad hoc networks in presence of channel randomness, IEEE Proc. of INFOCOM, Vo. 1, 13-17 pp. 491 - 502 Mar. 2005.

[8] M. D. Penrose, "On k-connectivity for a geometric random graph," Random Structures and Algorithms, vol. 15, no. 2, pp. 145-164, 1999.

[9] M. D. Penrose, "Extremes for the minimal spanning tree on normally distributed points," Advances in Applied Probability, vol. 30, no. 3, pp. 628-639, 1998.

[10] Peng-Jun Wan and Chih-Wei Yi, "Asymptotic critical transmission radius and critical neighbor number for $\mathrm{k}$ connectivity in wireless ad hoc networks," in Proceedings of ACM MobiHoc '04. 2004, pp. 1-8, ACM Press.

[11] Christian Bettstetter, "On the connectivity of Ad Hoc networks," Computer Journal, vol. 47, no. 4, pp. 432-447, July 2004.

[12] Esa Hyytiä, Pasi Lassila, and Jorma Virtamo, "Spatial node distribution in the random waypoint mobility model with applications," submitted for publication, available at http: //www.netlab.tkk.fi/julkaisut/bib/, Mar. 2005.

[13] Christian Bettstetter, "On the Minimum Node Degree and Connectivity of a Wireless Multihop Network," ACM Proc. of MobiHoc, Jun. 2002.

[14] Sayandev Mukherjee and Dan Avidor, "Connectivity and Transmit-Energy Considerations between Any Pair of Nodes in a Wireless Ad Hoc Network Subject to Fading," IEEE TRANSACTIONS ON VEHICULAR TECHNOLOGY, VOL. 57, NO. 2, MARCH 2008

[15] L. Hanzo, C. H. Wong, and M. S. Yee, Adaptive Wireless Transceivers.New York: John Wiley and Sons, 2002.

[16] A.V. Babu and Mukesh Kumar Singh, "node isolation probability of wireless adhoc networks in Nakagami fading channel," International journal of Computer Networks \& Communications (IJCNC), Vol.2, March 2010

[17] K. N, Nagesh; D, Satyanarayana; Poojary, Nagesh; Ramiah, Chandrashekar; , "An analytical expression for kconnectivity probability of wireless ad hoc networks in presence of channel randomness," Wireless Information Technology and Systems (ICWITS), 2012 IEEE International Conference on , vol., no., pp.1-4, 11-16 Nov. 2012

[18] P. Santi and D. M. Blough. "The critical transmitting range for connectivity in sparse wireless ad hoc networks." IEEE Trms. on Mob. Camp.,vol. 2, no. I, pp. 25-39. Jan-Mar 2003.

[19] 0. Douse, P. Thiran. and M. Haler, "Connectivity in ad-hoc and hybrid networks." in Pmc. of IEEE INFOCOM. New York USA. 2002.

[20] A. Rajeswaran and R. Negi. "Capacity of power constrained ad-hoc networks." in Pmc. of IEEE INFOCOM. Hong Kong. 2004.

[21] C. Zhong, M. Matthaiou, A. Huang, and Z. Zhang, "On the sum rate of MIMO Nakagami-m fading channels with linear receivers," submitted to IEEE Trans. Wireless Commun., Nov. 2011.

[22]C. Zhong, K.-K. Wong, and S. Jin, "Capacity bounds for MIMO Nakagami-m fading channels," IEEE Trans. Signal Process., vol. 57, no. 9, pp. 3613-3623, Sept. 2009.

[23] C. Zhong, S. Jin, T. Ratnarajah, and K.-K. Wong, "On the capacity of non-uniform phase MIMO Nakagami-m fading channels," IEEE Commun. Lett., vol. 14, no. 6, pp. 536-538, June 2010.

[24]M. R. McKay, I. B. Collings, and A. M. Tulino, "Achievable sum rate of MIMO MMSE receivers: A general analytic framework," IEEE Trans. Inf. Theory, vol. 56, no. 1, pp 396-410, Jan. 2010.

[25] M. Nakagami, "The m-distribution-A general formula of intensity distribution of rapid fading," in Statistical Methods in Radio Wave Propagation, W. C. Hoffman Ed., pp. 3-36, Oxford, U.K.: Pergamon, 1960.

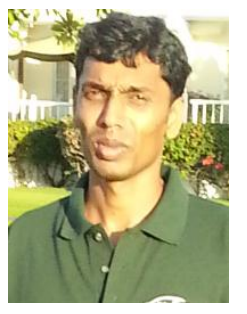

Nagesh K. Narayanaswamy obtained his Master degree in Digital electronics and communication from Visvesvaraya Technological University India, and Bachelor degree in Electronics \& Communication from Visvesvaraya Technological University India. His teaching interests are Communication systems, Digital communication. His research interest is Wireless communication. Currently he is working as a lecturer in the department of Electronics \& Communication at Middle East College, Muscat, Oman.

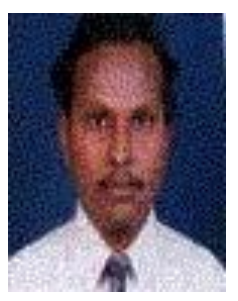

Satyanarayana D obtained his $\mathrm{PhD}$ from JNTU, India. His research interests are wireless networks and LP analysis. He has held various positions at university level. Currently he is working as Head of Electronics department at RGM College of Engineering and Technology, India.

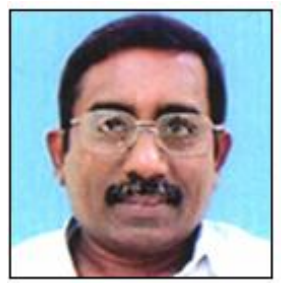

M.N Giri Prasad obtained his $\mathrm{PhD}$ from JNTU, India. His research interests are wireless network, communication systems. He has held various positions at university level. Currently he is working as Head of Electronics department at JNTUCE, Anantapur, India.

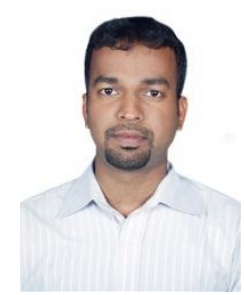

Madhava Prabhu S obtained his Master degree in Embedded Systems from Manipal University India, and Bachelor degree in Electronics \& Communication from Visvesvaraya Technological University India. His teaching interests are Microcontrollers, Digital logic design. His research interest are, Wireless communication, speech processing. Currently he is working as a lecturer in the department of Electronics \& Communication at Middle East College, Muscat, Oman. 